\title{
A Case Study of Wireless Sensor Network Latency in the Philippines for Enhancement of Packet-data Transmission
}

\author{
Felomino P. Alba ${ }^{+}$, Enrique D. Festijo and Ruji P. Medina \\ Technological Institute of the Philippines, Quezon City, Philippines
}

\begin{abstract}
Wireless sensor network is the engine of communication in the digital world. Communication rules the entire industry including business, medicine agriculture and many more. In this research paper, researchers discuss delay of packet data transmission of wireless sensor network communication in the Philippines. The research was conducted in a route based configuration wireless sensor network designed in a laboratory setting. Packet data congestion in network was identified by the researchers to be addressed. Openstat population-data of Philippine Statistic Authority were used during the simulation (http://openstat.psa.gov.ph/). Transmission delay of packet data from one route to another route was measured through response duration and return time-lapse. Researchers present an experimental evaluation of Dynamic Resource Routing (DRS), Self Selective Routing (SSR) and Ad-hoc Distance Vector Routing (ADVR). Experiments were evaluated to determine the lowest latency value of the returning node from a route going to next route. Authors compare different parameters such as number of nodes, source nodes, time interval, distance (meters ${ }^{2}$ ), stop-time, packet size and average number of wireless sensor users. Improvements to lessen the delay of transmission of packet data from devices passing through a multiple network route were listed. This research identifies the comparison of number of routes required to the number of users and the size of the packet data in a particular area in average. We proved that the number of source nodes (users) should have average number of route that correspond the lowering of network latency.
\end{abstract}

Keywords: wireless sensor network, network-latency, network layers, dynamic routing, ad-hoc, selective routing, data transmission

\section{Introduction}

Wireless sensor network (WSN) is a ad-hock network that are separated by distance elements and system configuration with protocol settings [1]. Wireless sensor network, a communication devices that transmits and received packet data, according to researchers [1] and [2]. Wireless sensor network is a communication of different devices that are separated by distance with system configuration, this can be in the domain of industrial, agriculture, automation, medical health, computer aided design, smart building and so on. [3] and [4]. An adhoc network that is deployed consists of small weighted sensor nodes in a physical environment with a sensor base in which the sensor nodes send all data collected. Wireless sensor network communications are temporal controlled decisions through a network layers [5]. In wireless communication, latency is one of the major concerns that exist in provisioning data centers which are geographically distributed [6]. The nodes between devices play a vital role that connects each one to communicate a certain volume of nodes and resulted to latency. Connectivity between computers to another computer is the simplest example of how network works. Sensor networks are expected to play an essential role in the upcoming age of pervasive computing [7].

However, when the nodes from one point of devices is sent through another devices, the response duration of the nodes creating a cycle or lapse time is the greatest key concepts of this research. Determining the delay in transmission of packet data from sending through the receiving end and the approaches best applied from either devices points or the route. Network latency of the connecting sensor nodes are the most

\footnotetext{
+ Corresponding author.

E-mail address: felominoalba@gmail.com
} 
efficient parameters on identifying the connectivity on multiple route on wireless sensor network. A typical network of computers connect with each other are controlled by different protocols applied. The connection that appears and present between the two devices on how and why they are connected is empowered by occurrences of different factors. One of the main factors is nodes latency communication

\section{Related Works}

\subsection{Wireless Sensor Network - Architecture}

According to authors [8], wireless sensor network now a days are the most common way of data links communication, mostly of the network devices were supported by wireless sensor network. Data packet broadcast is the fundamental function of wireless sensor networks and provides effective policy in all packet data protocol of connections and network topology. In a duty cycled network designed, network sensor nodes can only receives data on a allocated active time slot. Designing a free collision network makes more difficult for network designer for creating broadcast operations. [9], [10] Adhoc network contains large number of nodes that are governs by micro sensor that transmit and collect links autonomously in a multihop design gateway are wireless sensor network. Facility application surveillance, intrusion detection, industrial processes controls and machine health monitoring were among many applications used in wireless sensor network. [10] Wireless sensor network and mobile network technology are continuously derived in local network behavior performances [11].

\subsection{Network Broadcast Latency}

In [12] the authors found out that, multipath communication does not give advantages to application increase or decrease in terms of latency using paths multiple asymmetric, but could benefit from other advantages of the multipath technique. Sum of the delays in the communication path is understood as network latency according to [13]. In a time critical applications and scenarios, it is now widely used to understand network latency by [14].

\subsection{Data Delivery Latency}

In [15] latency minimization instead of routes length minimization is an efficient distribution system, the authors research resulted to delivery latency was minimized. In [16] models in adhoc wireless sensor network is the focus on the researchers, multi population parallel genetic algorithm in optimizing the structure and block based system latency. [17]. In [18] Gossip algorithms is a common term to describe protocols for unreliable information dissemination in natural networks, which are not optimally designed for efficient communication between network entities.

\section{Conceptual Framework}

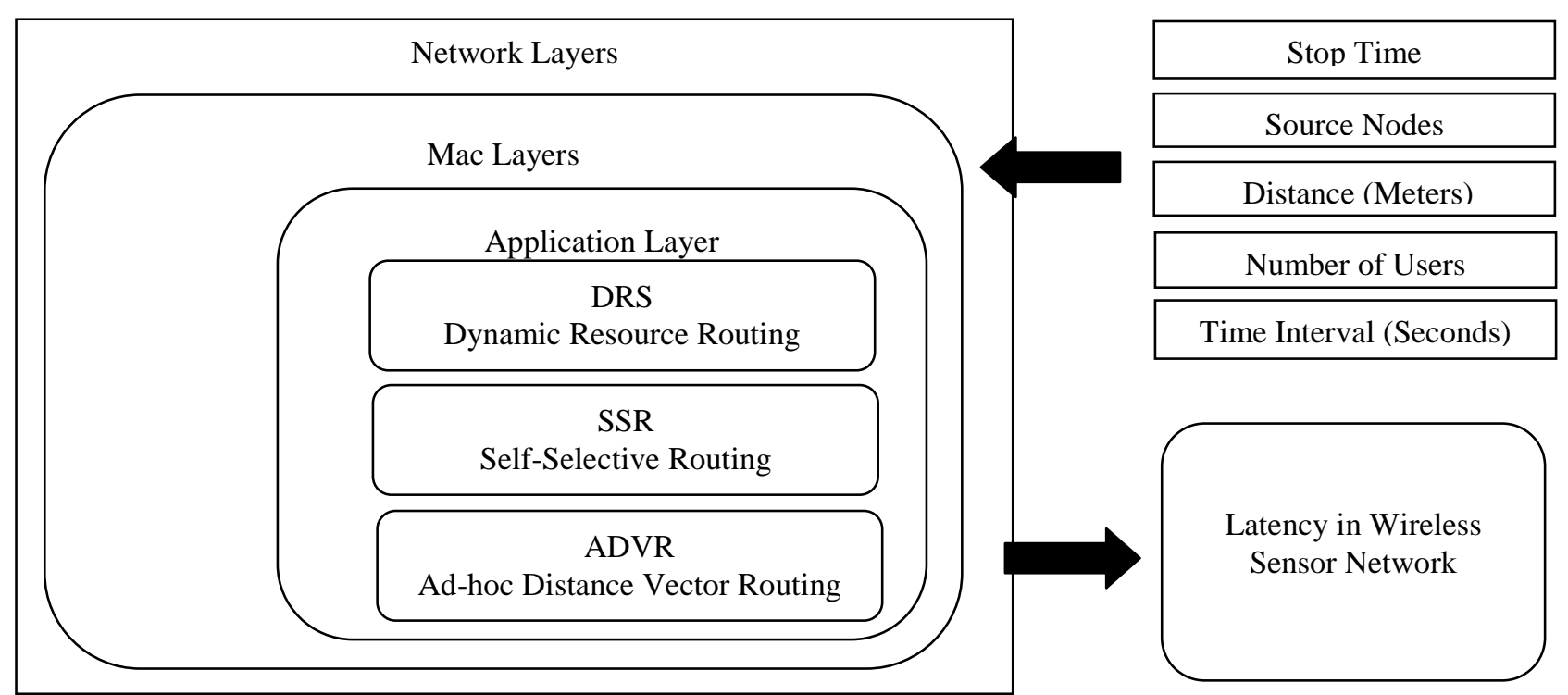

Fig. 1: Conceptual framework. 
Figure 1.0 presents the Conceptual Framework. The inputs are Stop Time, Number of Nodes, and Terrain Size in Meters, Number of Source of Nodes, Packet Size and Time Interval by Seconds. The process clearly defines the application of appropriate algorithm to come-up with desired output. These figures also illustrate the entire Operation Framework undertaken on this research paper. Simulation was conducted using the cygwin and g-sense simulator.

Table 1: Population selection.

\begin{tabular}{lcc}
\hline \hline Component City & Region & Population Density \\
\hline City of San Pedro, Laguna & 4 & 13,547 \\
Bacoor, Cavite & 4 & 13,009 \\
Cavite City, Cavite & 4 & 9,440 \\
City of Biñan, Laguna & 4 & 8,270 \\
City of Dasmariñas, Cavite & 4 & 7,312 \\
Cabuyao City, Laguna & 4 & 7,130 \\
City of Meycauayan, Bulacan & 3 & 6,513 \\
City of Santa Rosa, Laguna & 4 & 6,451 \\
City of Talisay, Cebu & 7 & 5,710 \\
City of San Jose del Monte, Bulacan & 3 & 5,440 \\
\hline
\end{tabular}

Table 1 shows the population selection of cities / municipalities including the regions. Top ten (10) most densely populated component cities in the Philippines in terms of sq.km based on Philippines Statistics Authority - Openstat data. Top ten (10) highest numbers of wireless sensor users.

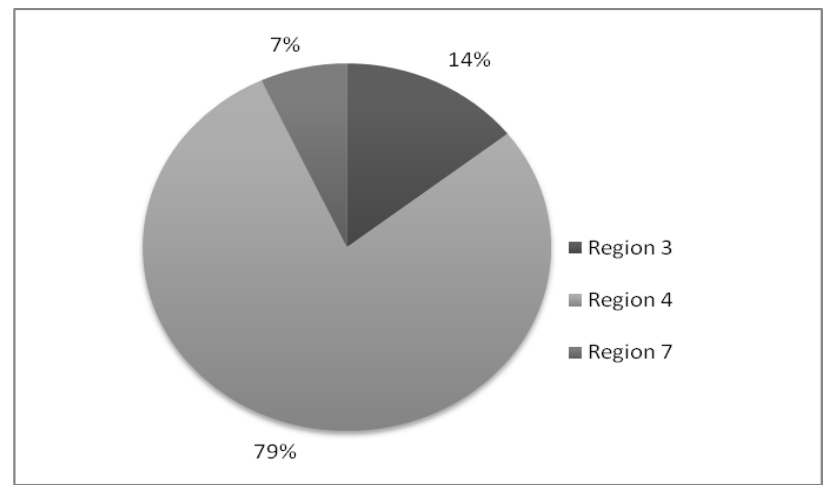

Fig. 2: Population density - wireless sensor network.

Table 2: Evaluation parameters 50 Users

\begin{tabular}{lcccccc}
\hline \hline Parameter & SanPedro & Bacoor & Cavite & Biñan & Dasmariñas & Cabuyao \\
\hline Stop Time & 1 & 1 & 1 & 1 & 1 & 1 \\
Terrain Size $\left(\mathrm{M}^{2}\right)$ & 1200 & 1200 & 1200 & 1200 & 1200 & 1200 \\
Node & 16 & 16 & 16 & 16 & 16 & 16 \\
Packet Size $(\mathrm{kb})$ & 128 & 128 & 128 & 128 & 128 & 128 \\
Interval $(\mathrm{s})$ & 2 & 2 & 2 & 2 & 2 & 2 \\
User's & 50 & 50 & 50 & 50 & 50 & 50 \\
\hline
\end{tabular}

Table 2 presents the Evaluation parameters of fifty (50) numbers of users, required for the simulation of response duration. Stop time and interval are in second's value. Packet-size was defined as 128kbyte for Global System for Messaging (GSM).

Table 3: Evaluation parameters 25 Users

\begin{tabular}{lcccccc}
\hline \hline Parameter & SanPedro & Bacoor & Cavite & Biñan & Dasmariñas & Cabuyao \\
\hline Stop Time & 1 & 1 & 1 & 1 & 1 & 1 \\
Terrain Size $\left(\mathbf{M}^{2}\right)$ & 2000 & 2000 & 2000 & 2000 & 2000 & 2000 \\
Node & 15 & 15 & 15 & 15 & 15 & 15 \\
Packet Size & 128 & 128 & 128 & 128 & 128 & 128 \\
Interval & 2 & 2 & 2 & 2 & 2 & 2 \\
User's & 25 & 25 & 25 & 25 & 25 & 25 \\
\hline
\end{tabular}


Table 3 presents the Evaluation parameters of twenty (25) number of users, required for the simulation of response duration. Stop time and interval are in second's value.

Table 4: Evaluation parameters

\begin{tabular}{lcccccc}
\hline \hline Evaluation & SanPedro & Bacoor & Cavite & Biñan & Dasmariñas & Cabuyao \\
\hline Dynamic Resource Routing & 50 & 50 & 50 & 50 & 50 & 50 \\
Self Selective Routing & 25 & 25 & 25 & 25 & 25 & 25 \\
Ad-hoc Distance Vector Routing & 50 & 50 & 50 & 50 & 50 & 50 \\
\hline
\end{tabular}

Table 4 - Present the Evaluation Parameters form table 1 with selected number of users.

\section{Result and Discussion}

Dynamic Resource Routing

\begin{tabular}{lcc}
\hline \hline Nodes & Position X & Position Y \\
\hline 0 & 242.6902 & 17.44876 \\
1 & 901.4589 & 620.0987 \\
2 & 1047.878 & 275.9362 \\
3 & 473.186 & 215.7369 \\
4 & 581.5455 & 490.5248 \\
5 & 64.46809 & 435.4343 \\
6 & 976.3832 & 612.0171 \\
7 & 632.8764 & 363.3609 \\
8 & 288.4824 & 301.919 \\
9 & 28.8735 & 1016.615 \\
10 & 328.4202 & 993.8756 \\
11 & 492.7825 & 739.0608 \\
12 & 399.974 & 16.75674 \\
13 & 780.633 & 137.9107 \\
14 & 659.1481 & 751.9807 \\
15 & 819.849856 & 324.782317 \\
\hline
\end{tabular}

Table 3 - Present the simulation Result on Dynamic Resource Routing of 50 users with 16 source nodes within 2,000 meters ${ }^{2}$ in each selected cities in table 1.

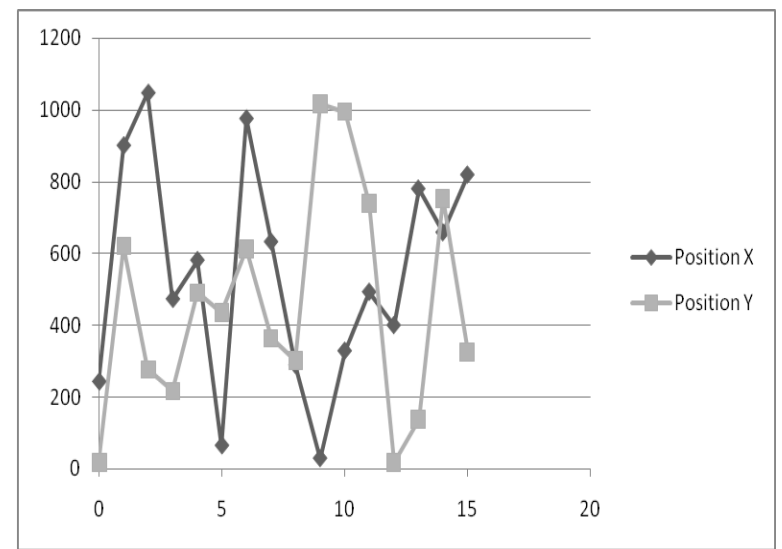

Fig. 3: Average delay comparisons on dynamic resource routing for 50 and 25 users with 16 source nodes.

Self Selective Resource Routing

\begin{tabular}{lcc}
\hline \hline Nodes & Position X & Position Y \\
\hline 0 & 485.380457 & 34.897 \\
1 & 1102.917967 & 1240.196 \\
2 & 195.755444 & 551.872 \\
3 & 946.371979 & 431.478 \\
\hline
\end{tabular}




\begin{tabular}{lcc}
\hline 5 & 128.936173 & 852.868 \\
6 & 1152.766639 & 1224.034 \\
7 & 1165.752781 & 726.721 \\
8 & 576.964865 & 603.837 \\
9 & 57.747002 & 2033.882 \\
10 & 656.840324 & 1187.751 \\
11 & 985.564953 & 1178.121 \\
12 & 799.968022 & 33.513 \\
13 & 1161.266028 & 275.821 \\
14 & 1118.296207 & 1503.961 \\
15 & 1139.688712 & 649.564 \\
\hline
\end{tabular}

Table 5 - Present the simulation Result on Self-Selective Resource Routing of 50 users with 16 source nodes within 2,000 meters $^{2}$ in each selected cities in table 1 .

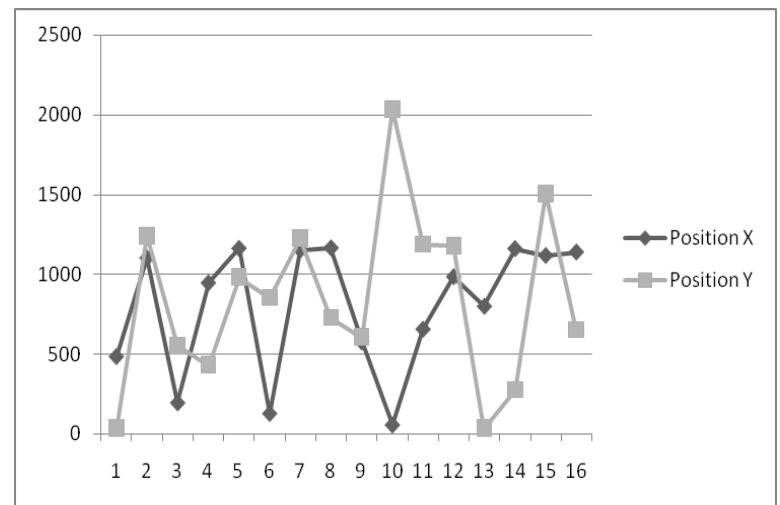

Fig. 4: Average delay comparisons on self-selective resource routing for 50 and 25 users with 16 Source Nodes.

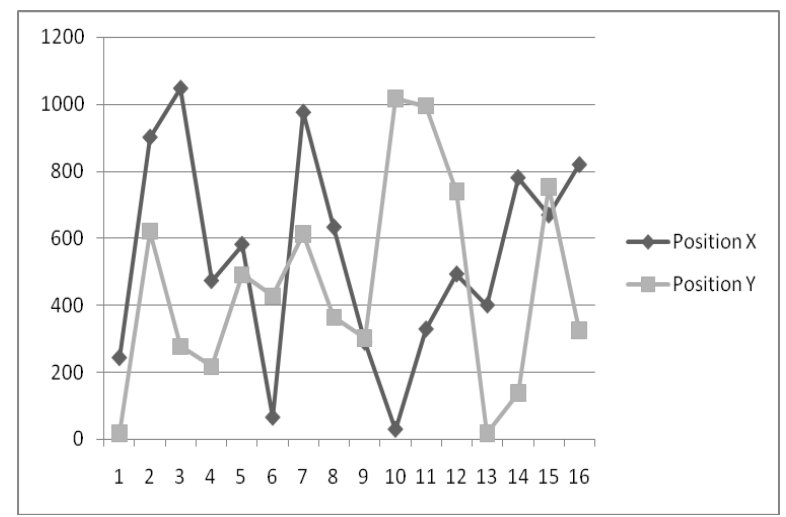

Fig. 5: Average delay comparisons on Ad-hoc distance vector routing for 50 and 25 users with 16 Source Nodes.

The researchers concluded based on the data simulated and the results that: The nodes are affected on delay while being transmitted especially with longer distance. The effects of distance to latency configuration are a great factor that determined lesser time delay. While connection between nearer route is much lesser in time delay, conclude to put up much closer route that connects the entire multiple nodes. The simulation purposes are to create a real time scenario to improved wireless sensor network settings and configuration in the Philippines. All data presented are free of use and it was based on PSA data available at (http://openstat.psa.gov.ph/search/type/dataset).

\begin{tabular}{lcc}
\hline \hline Criterion / Indicators & 50 users & 25 Users \\
\hline Application Layer & $33 \%$ & $2.5 \%$ \\
Network Layer & 0.210951 & 0.168913 \\
\hline
\end{tabular}




\begin{tabular}{lcc}
\hline MAC-Layers & 0.123749 & 0.116467 \\
Latency / Delay & 0.442 & 0.408 \\
\hline
\end{tabular}

\section{Recommendation}

Researchers recommend conducting further researches on the following topics: Time Delay interval that interferes on connecting devices security. Cryptography in wireless sensor network applies to transmission and receiving of packet-data. Wireless sensor network model and normal forms.

\section{References}

[1] K. Maraiya, K. Kant, and N. Gupta, "Wireless Sensor Network: A Review on Data Aggregation,” Int. J. Sci. Eng. Res., vol. 2, no. 4, pp. 1-6, 2011.

[2] D. Kim, B. H. Abay, R. N. Uma, W. Wu, W. Wang, and A. O. Tokuta, "Minimizing data collection latency in wireless sensor network with multiple mobile elements," INFOCOM, 2012 Proc. IEEE, pp. 504-512, 2012.

[3] K. CHELLI, "Security Issues in Wireless Sensor Networks," Proc. World Congr. Eng., vol. 1, 2015.

[4] D. V Queiroz et al., "Author's Accepted Manuscript Wireless Sensor Networks Reference :," J. Netw. Comput. Appl., 2017.

[5] L. Louail and V. Felea, "Latency optimization through routing-aware time scheduling protocols for wireless sensor networks," Comput. Electr. Eng., vol. 56, pp. 418-440, 2016.

[6] Y. Fan, H. Ding, L. Wang, and X. Yuan, "Green latency-aware data placement in data centers," Comput. Networks, vol. 110, pp. 46-57, 2016.

[7] A. Perrig, J. A. Stankovic, and D. Wagner, "Security in Wireless Sensor Networks," Commun. ACM, vol. 47, no. 6, pp. 53-57, 2004.

[8] J. A., K. R. S.V., and A. U. R., "Congestion avoidance algorithm using ARIMA(2,1,1) model-based RTT estimation and RSS in heterogeneous wired-wireless networks," J. Netw. Comput. Appl., vol. 93, pp. 91-109, 2017.

[9] D. T. Le, T. Le Duc, V. V. Zalyubovskiy, D. S. Kim, and H. Choo, "LABS: Latency aware broadcast scheduling in uncoordinated Duty-Cycled Wireless Sensor Networks," J. Parallel Distrib. Comput., vol. 74, no. 11, pp. 31413152, 2014.

[10] B. Neggazi, M. Haddad, and V. Turau, "A self-stabilizing algorithm for edge monitoring in wireless sensor networks," Inf. Comput., vol. 1, pp. 1-10, 2016.

[11] M. S. S. Khan, A. Kumar, B. Xie, and P. K. Sahoo, "Network Tomography Application in Mobile Ad-Hoc Network using Stitching Algorithm,” J. Netw. Comput. Appl., 2015.

[12] K. Yedugundla et al., "Is multi-path transport suitable for latency sensitive traffic?," Comput. Networks, vol. 105, pp. 1-21, 2016.

[13] O. Tomanek, P. Mulinka, and L. Kencl, "Multidimensional cloud latency monitoring and evaluation," Comput. Networks, vol. 107, no. Part 1, pp. 104-120, 2016.

[14] C. Ma, W. Liang, and M. Zheng, "Set-covering-based algorithm for delay constrained relay node placement in Wireless Sensor Networks," 2016 IEEE Int. Conf. Commun. ICC 2016, 2016.

[15] M. Moshref-Javadi and S. Lee, “The Latency Location-Routing Problem,” Eur. J. Oper. Res., vol. 255, no. 2, pp. 604-619, 2016.

[16] S. Nikoletseas, T. P. Raptis, and C. Raptopoulos, "Radiation-constrained algorithms for Wireless Energy Transfer in Ad hoc Networks," Comput. Networks, vol. 124, pp. 1-10, 2017.

[17] V. P. Nambiar, M. Khalil-Hani, M. N. Marsono, and C. W. Sia, "Optimization of structure and system latency in evolvable block-based neural networks using genetic algorithm," Neurocomputing, vol. 145, pp. 285-302, 2014.

[18] M. Siomau, "Gossip algorithms in quantum networks," Phys. Lett. A, vol. 381, no. 3, pp. 136-139, 2017. 